\title{
In vitro analysis reveals necroptotic signaling does not provoke DNA damage or HPRT mutations
}

\author{
Mark A. Miles (10 ${ }^{1}$ and Christine J. Hawkins (10
}

\begin{abstract}
Most anticancer drugs provoke apoptotic signaling by damaging DNA or other means. Genotoxic therapies may enhance a patient's risk of developing "therapy-related cancers" due to the accumulation of oncogenic mutations that may occur in noncancerous cells. Mutations can also form upon apoptotic signaling due to sublethal caspase activity, implying that apoptosis activating drugs may also be oncogenic. Necroptosis is a different way of killing cancer cells: this version of caspase-independent cell death is characterized by receptor-interacting protein kinase-3 (RIPK3) and mixed lineage kinase-like domain protein (MLKL) activation, leading to cell membrane rupture and controlled cell lysis. The mutagenic potential of sublethal necroptotic signaling has not yet been directly investigated. Smac mimetics drugs, which activate apoptotic or necroptotic cell death, do not induce mutations but the mechanistic basis for this lack of mutagenic activity has not been determined. In this study, we compared the mutagenic potential of these two cell death pathways by engineering cells to activate either apoptotic or necroptotic signaling by exposing them to Smac mimetics with or without TNFa, and/or enforcing or preventing expression of apoptotic or necroptotic regulators. We discovered that sublethal concentrations of Smac mimetics in contexts that activated apoptotic signaling provoked DNA damage and mutations in surviving cells. Mutagenesis was dependent on executioner caspase activation of the nuclease CAD. In contrast, RIPK3- and MLKL-dependent necroptotic signaling following Smac mimetic treatment was not mutagenic. Likewise, DNA damage was not provoked in cells expressing a lethal constitutively active MLKL mutant. These data reveal that cells surviving sublethal necroptotic signaling do not sustain genomic damage and provide hope for a reduced risk of therapy-related malignancies in patients treated with necroptosis-inducing drugs.
\end{abstract}

\section{Introduction}

Conventional chemotherapy drugs induce DNA damage and genotoxic stress by generating DNA crosslinks, adducts or strand breaks to hopefully initiate a DNA damage response leading to p53-mediated intrinsic apoptosis of malignant cells ${ }^{1}$. Some clinically employed and experimental anticancer agents such as $\mathrm{BH} 3$ mimetics or death ligands directly trigger proapoptotic functions by deactivating the prosurvival properties of Bcl-2-like proteins or activating death receptor-mediated extrinsic apoptotic signaling, respectively ${ }^{2,3}$. Unfortunately, DNA

Correspondence: Mark A. Miles (m.miles@latrobe.edu.au)

'Department of Biochemistry and Genetics, La Trobe Institute for Molecular Science, La Trobe University, Victoria, Australia

Edited by L. Sun damaging therapies can introduce mutations in cells that fail to die and mis-repair their DNA, with drugs exhibiting varied mutagenic potential depending on the type of lesions generated ${ }^{4}$. Such therapies can therefore spur the formation of second malignancies, known as "therapyrelated" cancers, in cured ex-patients particularly in individuals with germline defects that compromise the recognition or repair of DNA damage ${ }^{5,6}$. Furthermore, direct activation of apoptotic pathways via sublethal caspase activity can also be mutagenic ${ }^{7-9}$. Cleavage of ICAD by executioner caspases-3/7 enables the release and activation of the nuclease $C A D^{10}$, which generates double stranded DNA breaks that are recognized by the cell's DNA damage response system ${ }^{11,12}$. The mis-repair of CAD-mediated DNA strand breaks in cells that avoid a 
cell death fate following caspase activation highlights the mutagenic and potentially oncogenic consequence of sublethal apoptotic signaling ${ }^{13}$.

Inhibitor of apoptosis proteins (IAPs) are a class of prosurvival proteins that are often overexpressed in cancers, rendering many tumors resistant to chemotherapies ${ }^{14}$. Compounds antagonizing the prosurvival properties of IAPs have been designed to imitate the $\mathrm{N}$-terminal region of the cellular Smac/Diablo protein and as such these "Smac mimetics" display high affinity for cellular IAP 1 and 2 (cIAP1/2) and/or X-linked IAP (XIAP), but may also target other IAPs with low affinity ${ }^{15}$. Safe dosing regimens for a number of these compounds including LCL161, GDC0152, AT406 and Birinapant as single agents or in combination with other therapeutics have been determined, and subsequent clinical trials are ongoing ${ }^{16-20}$. Smac mimetics can activate two main forms of cell death: apoptosis or necroptosis. These drugs promote the auto-degradation of cIAP1/2, which normally act as E3 ubiquitin ligases to polyubiquitinate receptor-interacting protein kinase-1 (RIPK1) and establish NFkB activation of prosurvival pathways upon TNF receptor 1 (TNFR-1) ligation ${ }^{21}$. Nonubiquitinated RIPK1 can then interact with FADD to activate pro-caspase-8, which stimulates executioner caspase activity and thus apoptosis. Noncanonical NFkB-mediated TNF $\alpha$ production can also occur in some cell types following cIAP1/2 degradation to provide autocrine TNFR-1 stimulation and enhanced cell death propagation ${ }^{22}$. XIAP is a potent caspase- $3,-7$ and -9 inhibitor and mimetics can also relieve XIAP-mediated suppression of these proteases to allow apoptosis execution ${ }^{23,24}$. Conversely, necroptosis can be initiated in cells deficient in caspase-8 function. In this context, RIPK1 associates with its homolog receptorinteracting protein kinase-3 (RIPK3) via their RHIM domains to form the necrosome complex, activating RIPK3 which phosphorylates the pseudokinase MLKL (mixed lineage kinase domain-like protein) ${ }^{25}$. Active MLKL oligomerizes and translocates to the cell surface where plasma membrane pores are formed leading to the execution of necroptotic cell lysis ${ }^{26,27}$.

While necroptosis induction in vitro has been well characterized to require a concoction of TNF $\alpha$, Smac mimetic (to deplete cIAP1/2) and a caspase- 8 inhibitor, the activation of other death receptors, Toll-like receptors, or RHIM-domain-containing proteins have also been described to mediate this mode of cell death ${ }^{28}$. The utility of necroptosis in vivo has been proposed as an alternative mode of cell death to enable the destruction of apoptosisresistant pathogen infected cells, particularly to counteract the prosurvival action of viruses that suppress caspase8-mediated apoptosis ${ }^{29}$. As such, necroptosis is referred to as a pro-inflammatory form of caspase-independent cell death, evoking immune activation to aid in the clearance of compromised cells ${ }^{30}$.
Given the absence of apoptotic caspases in necroptotic cell death signaling, we reasoned that sublethal levels of necroptosis achieved would not mutate surviving cells, and may avoid the mutagenesis associated with direct DNA damage or sublethal apoptotic signaling. We manipulated cells to respond to Smac mimetics by either activating apoptotic or necroptotic pathways then assessed the induction of DNA damage and acquisition of mutations in clonogenically competent surviving cells. Mutagenesis at the hypoxanthine-guanine phosphoribosyltransferase (HPRT) locus was quantified by growing cells that received apoptotic or necroptotic stimuli in the presence of cytotoxic 6-thioguanine (6-TG), which suppresses growth of cells expressing functional HPRT but allows the proliferation and colony formation of HPRT defective cells due to drug-induced loss-of-function mutations $^{31}$. These cells are hence 6-TG resistant. Unlike Smac mimetic-induced apoptotic signaling, activation of RIPK3- and MLKL-dependent necroptotic signaling failed to provoke DNA damage or mutations in surviving cells.

\section{Materials and methods \\ Cell lines and reagents}

LN18 cells were purchased from ATCC, SV-40 transformed mouse embryonic fibroblasts (MEF) were kindly gifted by Anissa Jabbour and Paul Ekert, and U937 cells kindly gifted by James Murphy. LN18 and MEF cells were cultured in Dulbecco's modified Eagle medium with high glucose (Invitrogen; CA, USA), and U937 cells were cultured in RPMI-1640 containing HEPES buffer (Invitrogen). All media was supplemented with $10 \%$ FBS (Scientifix Life; VIC, Australia) and cells maintained at $37^{\circ} \mathrm{C}$ in air supplemented with $5 \% \mathrm{CO}_{2}$. LN18 cells stably expressing FLAG-MBP (clones 2.1 and 2.2) or FLAGCrmA (clones 1.4 and 1.5), or LN18 and U937 Cas9 control and CRISPR generated knockout cells deficient in MLKL (clones 4 and 31), RIPK3 (clones 9 and 13) or caspases-3/7 (C3/7 clones 2.5 and 1.7, or 2 and 8) have been previously described ${ }^{32}$.

The following drugs were used: AT406/Debio1143 (Selleck Chemicals; TX, USA), Birinapant (ApexBio; TX, USA), GDC0152 (Selleck), LCL161 (Selleck), soluble TRAIL (Peprotech; NJ, USA), doxycycline (Sigma; MO, USA), doxorubicin (Selleck) and cisplatin (Sigma). Other reagents include human and murine TNF $\alpha$ (Peprotech), Q-VD-OPh (R\&D Systems; MN, USA), necrostatin-1 (Sigma) and GW806742X (Sigma). The following antibodies were used: rabbit anti-phospho-MLKL Ser358 (Cell Signaling Technology; MA, USA; \#91689), rat antiMLKL clone 3H1 (gift from James Murphy), rabbit anti-RIPK3 (Cell Signaling Technology; \#13526), mouse anti-PARP (Cell Signaling Technology; \#9532), rabbit anti-phospho-H2AX Ser139 clone 20E3 (Cell Signaling 
Technology; \#9718), mouse anti-FLAG (M2) (Sigma; \#3165), mouse anti-GAPDH (Merck Millipore, MA, USA; MAB374), donkey anti-rabbit-HRP (GE Healthcare Life Sciences; NJ, USA; NA934), goat anti-rat-HRP (GE Healthcare Life Sciences; NA935), goat anti-rabbit-FITC (Merck Millipore; AQ132F), and rabbit anti-mouse-HRP (Sigma; A9044).

\section{Plasmids}

The pEF-FLAG-MBP ${ }^{11}$, pEF-FLAG-ICAD ${ }^{\text {D117, }}$ D224E (uncleavable ICAD mutant) $^{11}$ and pEF-FLAG-CrmA ${ }^{32}$ plasmids have been described previously. The pCW57GFP-2A-MCS was a gift from Adam Karpf (Addgene plasmid \#71783; http://n2t.net/addgene:71783; RRID: Addgene_71783) ${ }^{33}$. This plasmid was cut with BamHI and EcoRI, dephosphorylated and used to clone the following products in order to generate doxycycline inducible expression plasmids. Human RIPK3 coding sequence was amplified from U937 cDNA using primers: 5'-GCCA ATTGACCATGTCGTGCGTCAAGTTATGGCC- $3^{\prime}$ and 5'-GCAGATCTTTATTTCCCGCTATGATTATAC-3', and cut with MfeI and BglII. The N-terminal region (corresponding to the first 201 amino acids) of human MLKL was amplified using primers: $5^{\prime}$-GCGAATTCAC CATGGAAAATTTGAAGCATATTATC- $3^{\prime}$ and $5^{\prime}$-TT AAGATCTCTAAAGCTGCTCCTTCTTGATCTCC-3', and cut with EcoRI and BglII.

\section{Stable transfection}

Stable LN18 transfectants were generated using the FuGENE HD transfection reagent (Roche; Basel, Switzerland) as per manufacturer's instructions. After 2 days in fresh media, cells were selected in $3 \mu \mathrm{g} / \mathrm{ml}$ puromycin (Sigma) and colonies picked for characterization after 14-18 days. Stable U937 transfectants were generated by Nucleofection (Lonza; NJ, USA). One million U937 cells, $800 \mathrm{ng}$ of plasmid DNA and Nucleofector SF solution were combined then nucleofection performed using the DN-100 program on a Nucleofector device (Lonza). After 2 days growth in RPMI media containing 20\% FBS, cells were selected in $1 \mu \mathrm{g} / \mathrm{ml}$ puromycin by seeding at 1000 cells/well in round bottom 96-well plates and grown for 14-18 days before expanding for characterization.

\section{Cell death and cell survival}

Cell death was determined by flow cytometry. Cells were incubated with drugs or media for specified times then harvested and resuspended in binding buffer $(10 \mathrm{mM}$ HEPES, $140 \mathrm{mM} \mathrm{NaCl}, 2.5 \mathrm{mM} \mathrm{CaCl}$; $\mathrm{pH}$ 7.4) containing 1:200 of annexin-V-FITC (Abcam, Cambridge, UK) or annexin-V-V450 (BD Biosciences; CA, USA) depending on the experiment. Cells were incubated for $10 \mathrm{~min}$ at room temperature then an equal amount of binding buffer containing $2 \mu \mathrm{g} / \mathrm{ml}$ propidium iodide (Sigma) was added. Flow cytometric analysis was conducted using a FACS Canto II (BD Biosciences).

Clonogenic assays were performed to assess the ability of surviving cells to proliferate ${ }^{34}$. Following treatment, cells were washed once in PBS, counted and seeded at 100,300 , or 1000 cells per 6-well. Cells were stained with methylene blue (Sigma; $1.25 \mathrm{~g} / \mathrm{L}$ in $50 \%$ methanol) after 7 days for MEFs or 10-12 days for LN18 cells, and the number of colonies counted.

ATP activity in cells was measured using the CellTiterGlo 2.0 assay kit (Promega; WI, USA) to provide a readout of cell viability. Two thousand MEF or 10,000 U937 cells were seeded in white 96-well plates containing drugs or media and incubated for $24 \mathrm{~h}$. CellTiter-Glo reagent was added to wells at a ratio of 1:3 and plates incubated for $10 \mathrm{~min}$ at room temperature before luminescence detection. Luminescence was measured using a Spectromax M5e (Molecular Devices; CA, USA).

\section{Caspase activity assay}

DEVDase activity in cells was determined using the Caspase-Glo 3/7 assay kit (Promega). Two thousand LN18 or MEF cells, or 10,000 U937 cells were seeded in media alone or media containing drug into white 96-well plates and incubated for specified times. Caspase-Glo 3/7 reagent was added as 1:2 to each well and plates incubated at room temperature for $30 \mathrm{~min}$ prior to luminescence detection using a Spectromax M5e (Molecular Devices).

\section{pH2AX detection by flow cytometry}

Following drug treatment, cells were harvested and fixed in cold $70 \%$ ethanol at $-20^{\circ} \mathrm{C}$ overnight. The next day, ethanol was removed by centrifugation and cells rehydrated in TXT buffer (4\% FBS and 0.1\% Triton-X100 in TBS). Cells were stained with rabbit anti-phosphoH2AX Ser139 in TXT buffer (1:200) for $2 \mathrm{~h}$ shaking at room temperature, washed with TBS then stained with anti-rabbit-FITC antibody in TXT buffer (1:200) for $1 \mathrm{~h}$ shaking at room temperature. After washing with TBS, cells were finally resuspended in TBS containing $1 \mu \mathrm{g} / \mathrm{ml}$ propidium iodide and kept on ice until analysis of the FITC-positive signal of cells in G0/1 phase by a FACS Canto II (BD Biosciences).

\section{HPRT assay}

HPRT mutagenesis was determined by scoring the amount of colony growth in 6-thioguanine (6-TG; Sigma $)^{35}$. Ten (MEF) or 20,000 (LN18) cells were seeded in 24-wells a day before treatment. Following treatment, cells were washed with PBS and cultured for 7 days to ensure any residual HPRT enzymatic function was lost ${ }^{36}$, recounted with trypan blue to exclude dead cells then $3 \times$ $10^{5}$ cells seeded in media containing 6-TG $(1$ or $30 \mu \mathrm{M}$, MEF or LN18 cells respectively) in $15 \mathrm{~cm}$ culture dishes. 
Colonies were stained with methylene blue $(1.25 \mathrm{~g} / \mathrm{L}$ in $50 \%$ methanol) after 7 (MEF) or 18 (LN18) days.

\section{Immunoblotting}

Cells were lysed using RIPA lysis buffer $(150 \mathrm{mM}$ sodium chloride, $1.0 \%$ Triton $\mathrm{X}-100,0.5 \%$ sodium deoxycholate, $0.1 \%$ SDS, $50 \mathrm{mM}$ Tris, $\mathrm{pH}$ 8.0, supplemented with protease inhibitor cocktail; Sigma) by pipetting 50 times and rapid vortexing. The lysates were cleared by centrifuging for $15 \mathrm{~min}$ at $16,100 \mathrm{~g}$ at $4{ }^{\circ} \mathrm{C}$. Total protein was determined using the bicinchoninic acid (BCA) method (Micro BCA Protein assay kit, Thermo Fisher Scientific; MA, USA). Fifty micrograms of lysates were loaded on tris-glycine gels and the proteins were separated by sodium dodecyl sulfatepolyacrylamide gel electrophoresis then transferred onto Hybond PVDF $0.22 \mu \mathrm{m}$ membrane (Millenium Science; Victoria, Australia). Membranes were blocked with $1 \%$ blocking reagent (Roche) in phosphate-buffered saline (PBS), and probed with primary antibodies then horseradish peroxidase (HRP)-conjugated secondary antibodies diluted in $1 \%$ blocking reagent (Roche) in PBS with 0.1\% Tween-20 (Sigma). SuperSignal West Dura extended duration substrate (Thermo Fisher Scientific) was used for detection.

\section{Statistics}

GraphPad Prism 8.0 was used to perform one-way or two-way ANOVA analyses (specified in the figure legends) with Sidak post-tests to assess the significance of differences in responses of untreated and treated cells. All experiments were independently repeated three times and data presented as the mean value with error bars representing SEM from biological replicates.

\section{Results \\ Caspase-deficient cell death signaling does not provoke mutations}

We assayed three cell lines of different lineages to assess the ability of TNFo co-treatment with Smac mimetics to provoke DNA damage or mutate cells at the HPRT locus. Treatment of U937, MEF, or LN18 cells with TNF $\alpha$ combined with various Smac mimetics enabled annexin-V binding to phosphatidylserine and/or membrane permeabilization (Fig. 1a), enhanced DEVDase levels (Fig. 1b), and induced DNA damage as indicated by $\mathrm{H} 2 \mathrm{AX}$ phosphorylation $(\gamma \mathrm{H} 2 \mathrm{AX})$ (Fig. 1c). The addition of the pan caspase inhibitor Q-VD-OPh (QVD) suppressed DEVDase activity and the ensuing DNA damage following treatment in all cell types, implying DNA damage coincided with caspase activity, but only spared LN18 cells from death indicating caspase-independent death was achieved in U937 and MEF cells. Furthermore, $\mathrm{TNF} \alpha / \mathrm{Smac}$ mimetic signaling occurred via RIPK1 in
U937 and MEF cells as necrostatin-1 (Nec-1) reduced cell death and DEVDase activity in these cells. RIPK1 inhibition by Nec-1 also inhibited the DNA damage provoked by co-treatment with Smac mimetics plus TNFo.

Mutation assays were then performed to determine if the DNA damage we observed resulted in mutations at the HPRT locus, and whether the lack of $\gamma \mathrm{H} 2 \mathrm{AX}$ induction under conditions of caspase-independent cell death reflected a lack of mutagenesis. Incubation of LN18 or MEF cells with DNA damaging chemotherapy agents impaired clonogenicity and enabled colony formation in the presence of 6-TG (Supplementary Fig. 1), implying HPRT mutations and validating this assay. TNF $\alpha$ alone did not affect clonogenic survival or increase the frequency of 6-TG resistant cells but more colonies emerged when TNF $\alpha$ was combined with Smac mimetics implying that, unlike non-TNF $\alpha$-stimulated Smac mimetic exposure (Supplementary Fig. 1a), the combination treatment provoked HPRT mutations (Fig. 2). As expected, pretreatment with QVD protected LN18 cells from TNF $\alpha /$ Smac mimetic-induced clonogenic death and prevented the emergence of 6-TG resistant colonies (Fig. 2a). This effect was further observed in cells deficient in caspases-3 and $-7(\mathrm{C} 3 / 7 \mathrm{DKO})$ as these cells only displayed background levels of $\gamma \mathrm{H} 2 \mathrm{AX}$ induction or 6-TG resistant colonies following TNF $\alpha /$ LCL161 treatment (Supplementary Fig. 2). Interestingly, incubation with TNF $\alpha /$ LCL161 impaired clonogenicity of C3/7 DKO lines but to a lesser degree than Cas9 control (Supplementary Fig. 2b). The incomplete protection afforded by loss of the executioner caspases most likely reflects mitochondrial impairments upstream of executioner caspases that impacted on clonogenicity, rather than necroptosis as these cells lack RIPK3 expression ${ }^{32}$. TNF $\alpha$ combined with LCL161 also impeded the clonogenic potential of MEF cells in a dose dependent manner, and this effect was maintained even when caspases were inhibited by QVD (Fig. 2b). More 6-TG resistant colonies were formed from MEF cultures that survived TNF $\alpha / L C L 161$ exposure in the absence QVD pretreatment.

Prior research has described the ability of the caspase activated nuclease CAD to damage DNA and provoke mutagenesis upon apoptotic stimuli $78,11,37,38$. We stably expressed a mutant version of ICAD (mICAD), which bears glutamic acid residues at the $\mathrm{P} 1$ position of the caspase cleavage sites to render it uncleavable by active caspases ${ }^{10}$, in LN18 and U937 cells to confirm the ability of TNF $\alpha / L C L 161$ co-treatment to damage DNA via active CAD (Supplementary Fig. 3). Similar levels of cell death and caspase activation were achieved between all stable lines, while an increase in the number of cells bearing $\gamma \mathrm{H} 2 \mathrm{AX}$ after TNF $\alpha / \mathrm{LCL} 161$ treatment was only detected in MBP stable transfectants and not in cells expressing mICAD. 


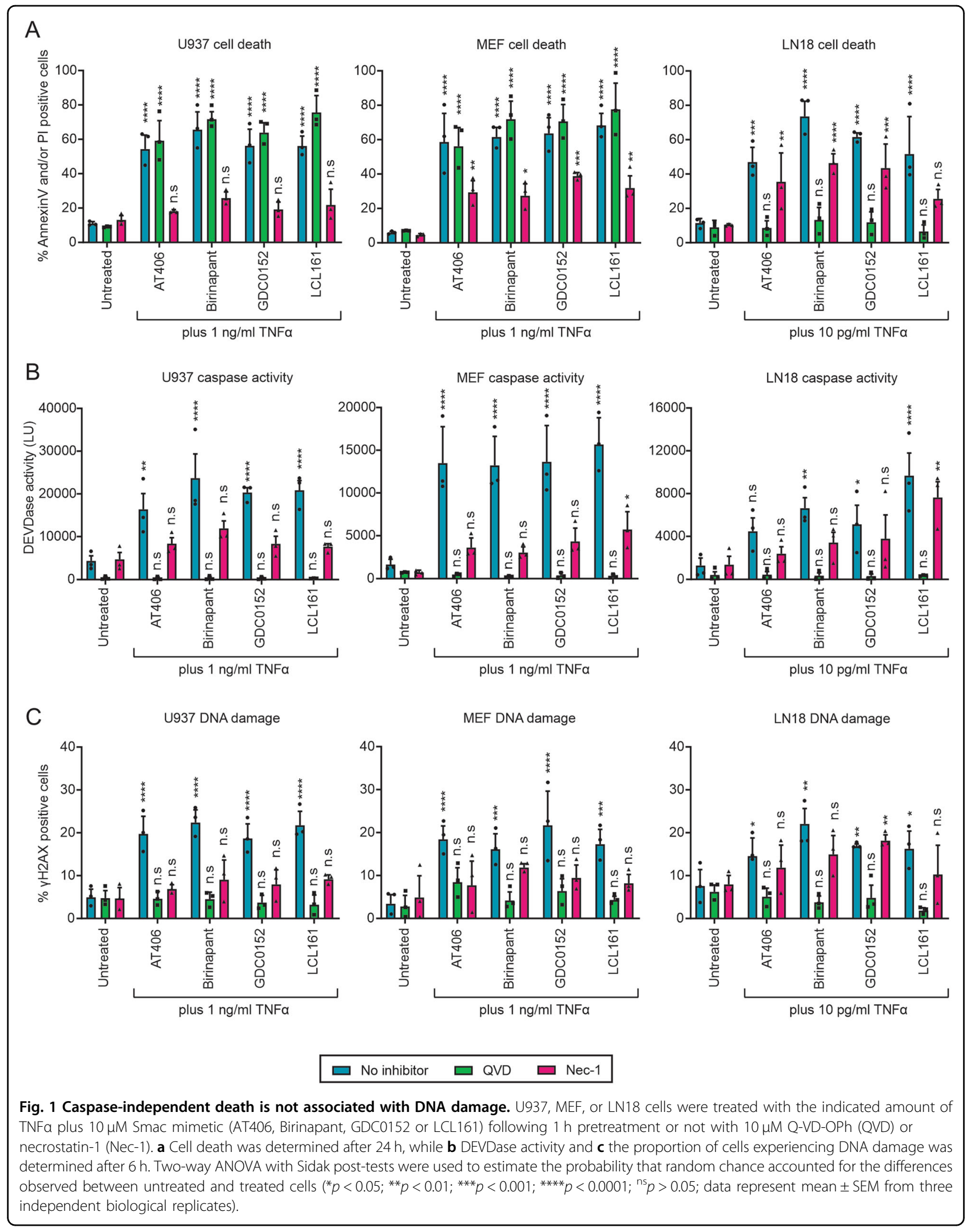



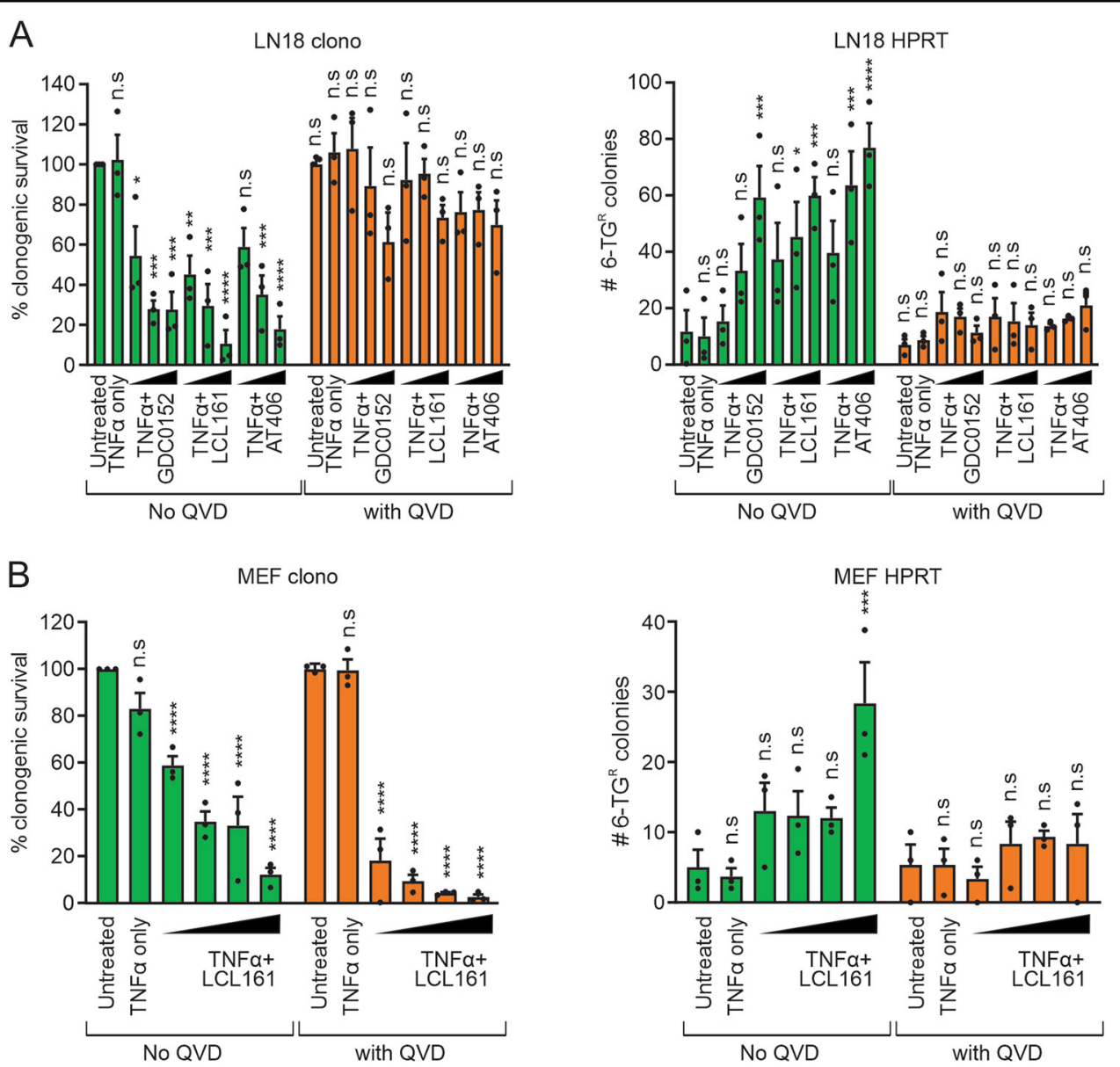

Fig. 2 Co-treatment of TNFa and Smac mimetics provoke HPRT mutations but only under caspase proficient conditions. a LN18 cells were incubated or not with Smac mimetics $(0.3,1$, or $3 \mu \mathrm{M})$ in the presence of $10 \mathrm{pg} / \mathrm{ml} \mathrm{TNFa}$, while $\mathbf{b}$ MEF cells were exposed to $1 \mathrm{ng} / \mathrm{ml}$ TNFa plus LCL161 $(0.3,1$, 3, or $10 \mu \mathrm{M})$. Some cells were pretreated with $10 \mu \mathrm{M}$ QVD. Following $24 \mathrm{~h}$, the clonogenic potential was determined (left panels) and the surviving cells grown in 6-TG to select for the emergence of HPRT mutant colonies (right panels). Two-way ANOVA with Sidak post-tests were used to estimate the probability that random chance accounted for the differences observed between untreated and treated cells $\left(^{*} p<0.05 ;{ }^{* *} p<\right.$ $0.01 ;{ }^{* *} p<0.001 ;{ }^{* * *} p<0.0001 ;{ }^{n 5} p>0.05$; data represent mean \pm SEM from three independent biological replicates).

These data reveal that cells surviving TNF $\alpha$ and Smac mimetic co-treatment acquire DNA damage and HPRT mutations via CAD-mediated DNA damage following activation by sublethal levels of executioner caspases, and that mutagenesis does not occur under caspase-deficient conditions.

\section{RIPK3 and MLKL are required for non-mutagenic necroptotic signaling}

Given that Smac mimetics have been documented to induce RIPK3/MLKL-mediated necroptotic cell death in cells that lack caspase- 8 activity and express RIPK $3^{39,40}$, we confirmed that necroptotic signaling was activated in U937 and MEF cells upon TNF $\alpha /$ LCL161/QVD treatment, and found that this did not promote mutagenesis. U937 cells deficient in either RIPK3 or MLKL $^{32}$ were insensitive to TNF $/ \mathrm{LCL} 161 / \mathrm{QVD}$-induced death
(Supplementary Fig. 4a), confirming classical RIPK3-/ MLKL-dependent necroptotic signaling was triggered by TNFR-1 ligation while IAPs and caspase- 8 were inhibited. GW806742X was used to confirm the requirement for MLKL in TNF $\alpha / L C L 161 / Q V D$-induced death in MEF cells, as this compound suppresses MLKL activity by targeting the pseudokinase domain of murine MLKL ${ }^{41}$. GW806742X maintained the viability of MEF cells treated with TNF $\alpha / L C L 161 / Q V D$ and (to a lesser extent) TNF $\alpha /$ LCL161 (Supplementary Fig. 4b). The slight protection of cells treated with TNF $/$ LCL161 could be due to chance or possibly due to minor affinity of the inhibitor for RIPK1 ${ }^{41}$.

Cells that were sensitive to necroptotic conditions (Cas9 or C3/7 deficient cells) did not exhibit H2AX phosphorylation, while an increase in cells experiencing DNA damage was observed only in cells that were proficient in 
caspase-3/7 activity and treated with TNFa/LCL161, regardless of RIPK3 or MLKL expression (Supplementary Fig. 4c). Phosphorylated H2AX was detected in TNF $\alpha /$ LCL161-treated lysates that also contained cleaved PARP, indicating caspase activation, but not in lysates of TNF $\alpha$ / LCL161/QVD-treated cells that contained phosphorylated (and therefore active) MLKL and lacked PARP cleavage (Supplementary Fig. 4d). These experiments confirm that TNF $/$ LCL161/QVD treatment activates MLKL-mediated necroptosis in U937 and MEF cells, and that this pathway does not damage DNA.

LN18 cells did not succumb to TNF $\alpha /$ Smac mimeticmediated cell death in the presence of QVD most likely because these cells do not express RIPK3 thereby prohibiting MLKL activation via the necrosome ${ }^{32,39}$. To sensitize LN18 cells to necroptosis, we engineered these cells to contain a doxycycline inducible RIPK3 expression plasmid. Cells capable of inducible GFP expression were used as a control. Non-lethal RIPK3 expression in LN18 cells was achieved when cells were grown in $1 \mu \mathrm{g} / \mathrm{ml}$ doxycycline (Fig. 3a). TNF $/$ LCL161 treatment of LN18 cells expressing RIPK3, or not, resulted in cleavage of PARP, cell death and enhanced DEVDase levels (Fig. 3a-c), indicating caspase-dependent death. As expected, phosphorylated $\mathrm{H} 2 \mathrm{AX}$ was also detected in these cells. GFP expressing cells treated with TNF $\alpha /$ LCL161 in the presence of QVD lacked PARP cleavage, remained viable (Fig. 3b) and did not display DEVDase activity (Fig. 3c) or DNA damage (Fig. 3a). In contrast, RIPK3 expressing cells treated under the same conditions displayed phosphorylation of RIPK3 (as indicated by the upper band in doublet ${ }^{42}$ ) and MLKL but not H2AX (Fig. 3a). RIPK3 expression also enabled the killing of LN18 cells by TNF $\alpha / L C L 161 / Q V D$ treatment (Fig. 3b) and this occurred in the absence of DEVDase activity (Fig. 3c). These data confirm the activation of necroptosis in RIPK3-expressing LN18 cells treated with TNF $\alpha / L C L 161 /$ QVD. Unlike GFP, inducible expression of RIPK3 impaired clonogenicity following TNF $\alpha / L C L 161 / Q V D$ treatment (Fig. 3d) and this was not associated with an increase in the number of 6-TG resistant colonies (Fig. 3e). Exposure to TNF $\alpha / \mathrm{LCL} 161$ in the absence of QVD provoked more 6-TG resistant colonies in GFP or RIPK3 expressing cells. This demonstrates that expression of RIPK3 was required to enable necroptotic signaling upon TNF $\alpha /$ LCL161 treatment in LN18 cells under caspase deficient conditions, and revealed that this signaling does not provoke DNA damage or mutations.

To directly link the non-mutagenicity of necroptotic signaling to MLKL-mediated death, we used the doxycycline inducible expression system to inducibly express a constitutively active $\mathrm{N}$-terminal region (corresponding to the first 201 amino acids) of human MLKL (MLKL ${ }^{1-201}$ ) in LN18 cells (Fig. 4a). This was published to activate necroptosis when over-expressed ${ }^{43}$. Expression of MLKL ${ }^{1-201}$ provoked annexin-V binding and uptake of propidium iodide over time, which was not accompanied by DEVDase activity or DNA damage (Fig. 4b-d). In contrast, treatment of inducible clones in the absence of doxycycline (therefore no expression of active MLKL $^{1-201}$ ) with TRAIL or cisplatin provoked an increase in the number of cells containing $\gamma \mathrm{H} 2 \mathrm{AX}$. TRAIL enabled annexin- $\mathrm{V}$ binding to phosphatidyl serine prior to propidium iodide uptake and provoked rapid DEVDase activity, whereas cells remained viable after cisplatin exposure at these early time points, consistent with its indirect toxic mechanism ${ }^{44,45}$.

\section{Necroptosis is non-mutagenic in the context of $\mathrm{CrmA}$ expression}

The data presented so far has utilized QVD to chemically inhibit caspase-8 (as well as other caspases) in order to shift TNFR-1 signaling from apoptosis to necroptosis. To confirm that necroptotic signaling was also not mutagenic under conditions whereby a chemical caspase inhibitor was not present, we stably expressed CrmA (a poxviral Spi2 protein that can efficiently inhibit caspase- $8^{46}$ ) in U937 and LN18 cells to incapacitate caspase- 8 and promote TNF $\alpha /$ LCL161-mediated necroptosis without the requirement for QVD addition (Fig. 5a). TNF $/$ LCL161 treatment provoked MLKL phosphorylation in U937 cells expressing CrmA but not in MBP expressing lines (Fig. 5b). Conversely, CrmA expression in LN18 cells did not enable phosphorylation of MLKL upon TNF $\alpha / L C L 161$ exposure due to a lack of basal RIPK3 expression ${ }^{32}$. U937 CrmA stable transfectants were killed by TNF $\alpha / L C L 161$ but, unlike MBP stable transfectants, this treatment was not accompanied by enhanced DEVDase activity nor did it increase the proportion of $\gamma \mathrm{H} 2 \mathrm{AX}$ positive cells (Fig. 5c). TNF $\alpha / \mathrm{LCL} 161$ treatment also failed to provoke $\mathrm{H} 2 \mathrm{AX}$ phosphorylation in LN18 CrmA stable transfectants (Fig. 5d). These cells also maintained clonogenic viability despite TNF $\alpha /$ LCL161 exposure and, unlike MBP-expressing cells, only background numbers of 6-TG resistant colonies were detected following treatment (Fig. 5e). These data indicate that inhibition of caspase- 8 by CrmA sensitizes U937 (but not LN18) cells to necroptosis induced by TNF $\alpha / L C L 161$, and CrmA expression in either cell type protects from the mutagenesis associated with TNF $\alpha /$ LCL161 treatment.

\section{Discussion}

Defining the molecular components and mechanisms of various modes of cell death has been a key focus in recent years to further understand disease, such as cancers and inflammatory disorders, with the intention of developing therapeutics to effectively manipulate these cell death pathways. This is emphasized by the need to effectively 


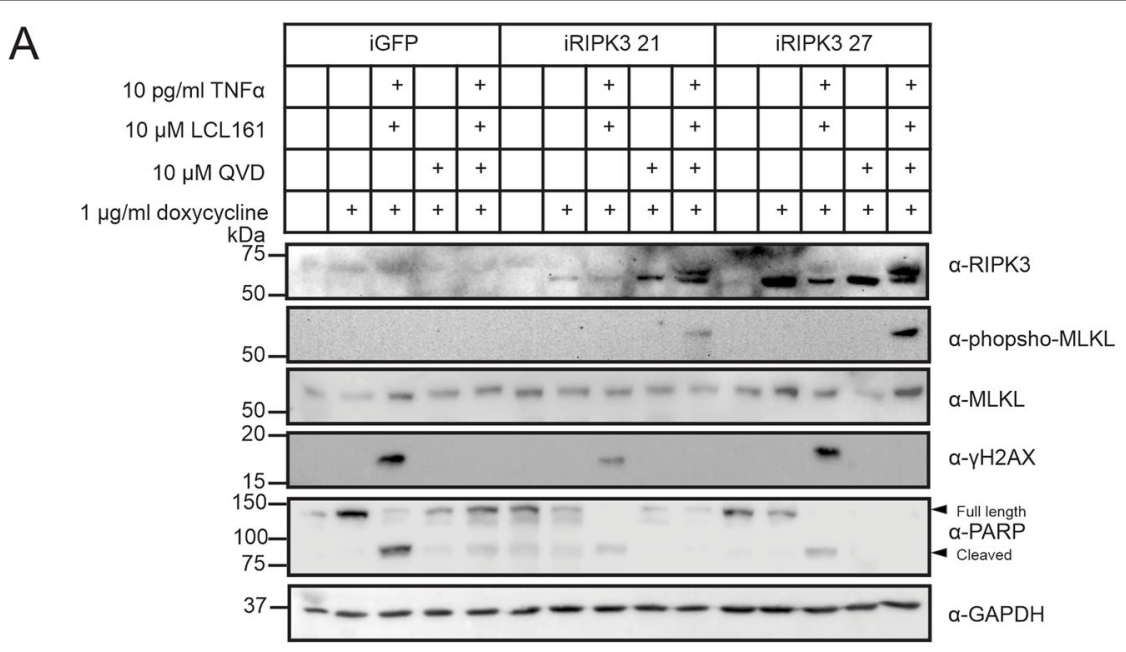

B

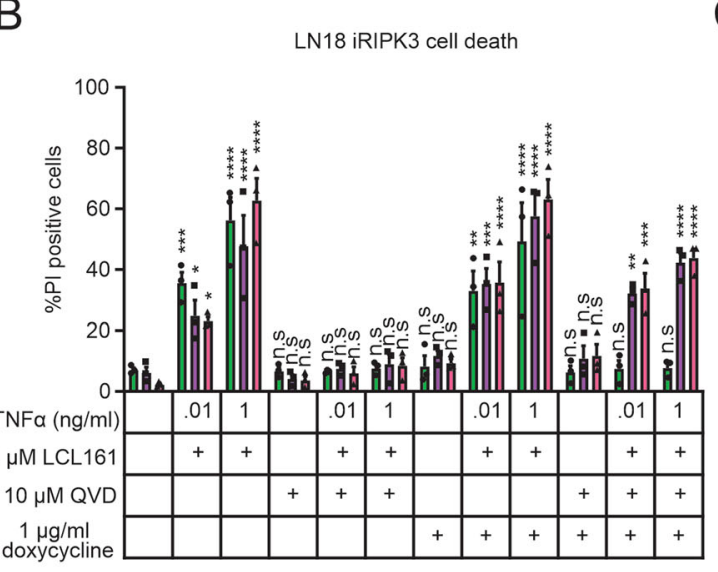

D

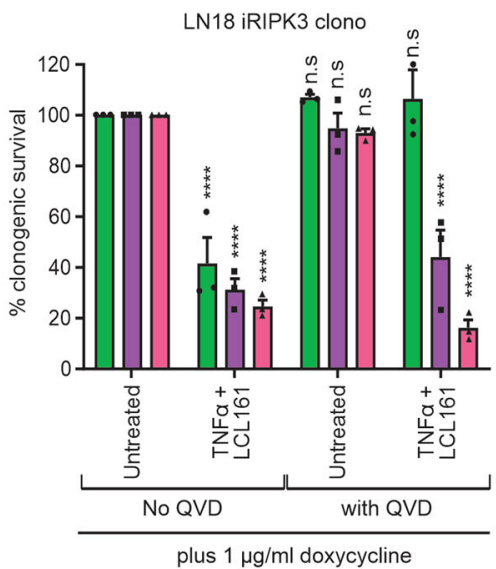

C

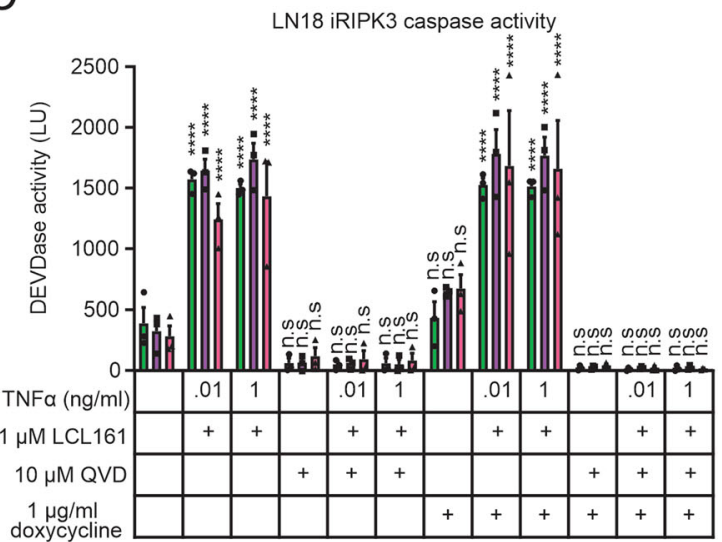

$\mathrm{E}$

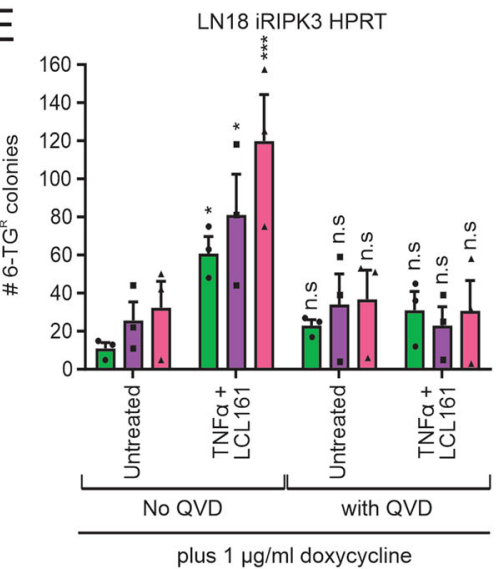

$\square$ iRIPK3 27

Fig. 3 Inducible expression of RIPK3 sensitizes LN18 cells to necroptosis which is not mutagenic. LN18 cells transfected with a GFP or RIPK3 inducible expression plasmid were grown in doxycycline (to induce expression) for $24 \mathrm{~h}$ prior to treatment, then treated with TNFa, LCL161, and/or QVD (1 h pretreatment). a Immunoblotting was performed to confirm RIPK3 expression and determine phosphorylated and total levels of MLKL, phosphorylated H2AX, full length and cleaved PARP, or GAPDH. b PI uptake was used to determine the proportion of dead cells and $\mathbf{c}$ DEVDase activity also measured. RIPK3 expressing cells treated with $10 \mathrm{pg} / \mathrm{ml}$ TNFa plus $1 \mu \mathrm{M} L \mathrm{LL} 161$, with $10 \mu \mathrm{M}$ QVD pretreatment or not, were assessed for $\mathbf{d}$ changes in clonogenic potential and $\mathbf{e}$ the ability of surviving cells to grow and form colonies in 6-TG. Two-way ANOVA with Sidak post-tests were used to estimate the probability that random chance accounted for the differences observed between untreated and treated cells $\left({ }^{*} p<0.05 ;{ }^{* *} p<\right.$ 0.01 ; ${ }^{* * *} p<0.001 ;{ }^{* * *} p<0.0001 ;{ }^{\text {ns }} p>0.05$; data represent mean \pm SEM from three independent biological replicates). 

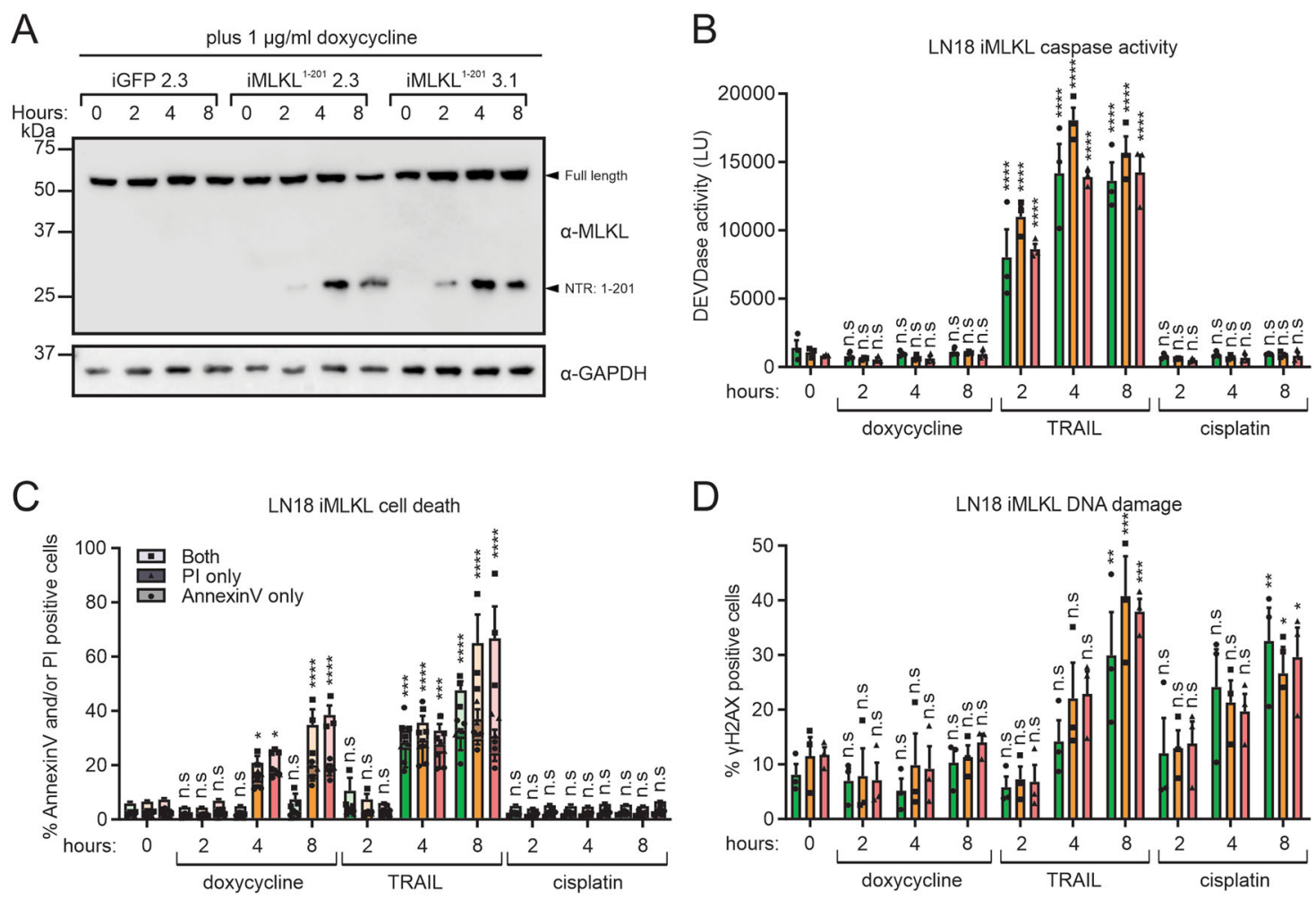

D

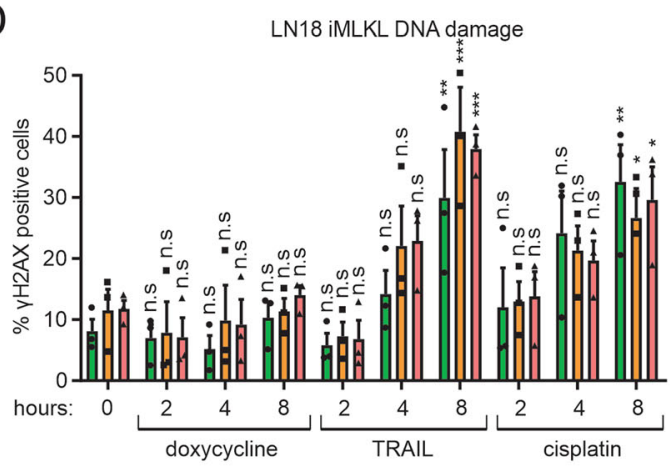

LN18 inducible clones: $\square$ iGFP $\square$ iMLKL $^{1-201} 2.3 \quad \square$ iMLKL $^{1-201} 3.1$

Fig. 4 Inducible MLKL activation does not provoke DNA damage. $L N 18$ cells transfected with a GFP or MLKL N-terminal region (MLKL ${ }^{1-201}$ ) inducible expression plasmid were grown in doxycycline (to induce expression) for the indicated time points. a Immunoblotting was used to confirm expression of $\mathrm{MLKL}^{1-201}$. Inducible clones were grown in $1 \mu \mathrm{g} / \mathrm{ml}$ doxycycline, $10 \mathrm{ng} / \mathrm{ml}$ TRAIL, or $1 \mu \mathrm{M}$ cisplatin for indicated time points. b DEVDase activity, $\mathbf{c}$ cell death and $\mathbf{d}$ proportion of cells experiencing DNA damage were determined. Two-way ANOVA with Sidak post-tests were used to estimate the probability that random chance accounted for the differences observed between untreated and treated cells $\left({ }^{*} p<0.05 ;{ }^{* *} p<0.01 ;{ }^{* * *} p\right.$ $<0.001 ;{ }^{* * * *} p<0.0001 ;{ }^{\text {ns }} p>0.05$; data represent mean \pm SEM from three independent biological replicates).

eliminate cancer cells that have acquired chemoresistance to apoptotic cell death provoked by many conventional chemotherapeutics ${ }^{47}$. The inaccurate repair of DNA lesions generated by genotoxic therapies can introduce mutations in non-cancerous cells to facilitate oncogenic transformation or enhance intratumoural heterogeneity in a subpopulation of apoptosis-resistant clones, thereby impacting on the formation of subsequent or relapsed cancers ${ }^{48,49}$. Understanding the mutagenic potential of different cell death signaling pathways that are activated by various anti-cancer therapies is therefore important as non-mutagenic pathways may reduce a patient's risk of therapy-related cancer. As knowledge of necroptotic signaling pathways improves, we sought to define the mutagenic potential of this caspase-independent cell death pathway and determine if cells acquire mutations after experiencing sublethal activation of these pathways.

Smac mimetics are commonly used in vitro to activate necroptotic pathways when combined with TNFa and an inhibitor that targets caspase-8, so we used this approach to specifically interrogate the mutagenic potential of necroptotic signaling. We observed that sublethal exposure to Smac mimetics without concurrent TNF $\alpha$ treatment was non-mutagenic ${ }^{34,50}$. We recently discovered that those treatments triggered a lytic, non-apoptotic and non-necroptotic mode of death ${ }^{32}$. In this study, we engineered scenarios in which cellular responses to Smac mimetics could be switched between apoptotic and necroptotic pathways, to determine the mutagenesis associated with sublethal activation of each cell death mechanism. We found that activation of classical apoptotic pathways in cells sensitive to TNF $\alpha$ and Smac mimetic co-treatment provoked mutations in surviving cells that maintained clonogenic competency. Using QVD to inhibit caspase activity, cells lacking executioner caspase-3/7 expression, or stable expression of a noncleavable mutant form of ICAD, we confirmed that the mutagenesis observed occurred via the mis-repair of DNA damage generated by the CAD nuclease upon sublethal 


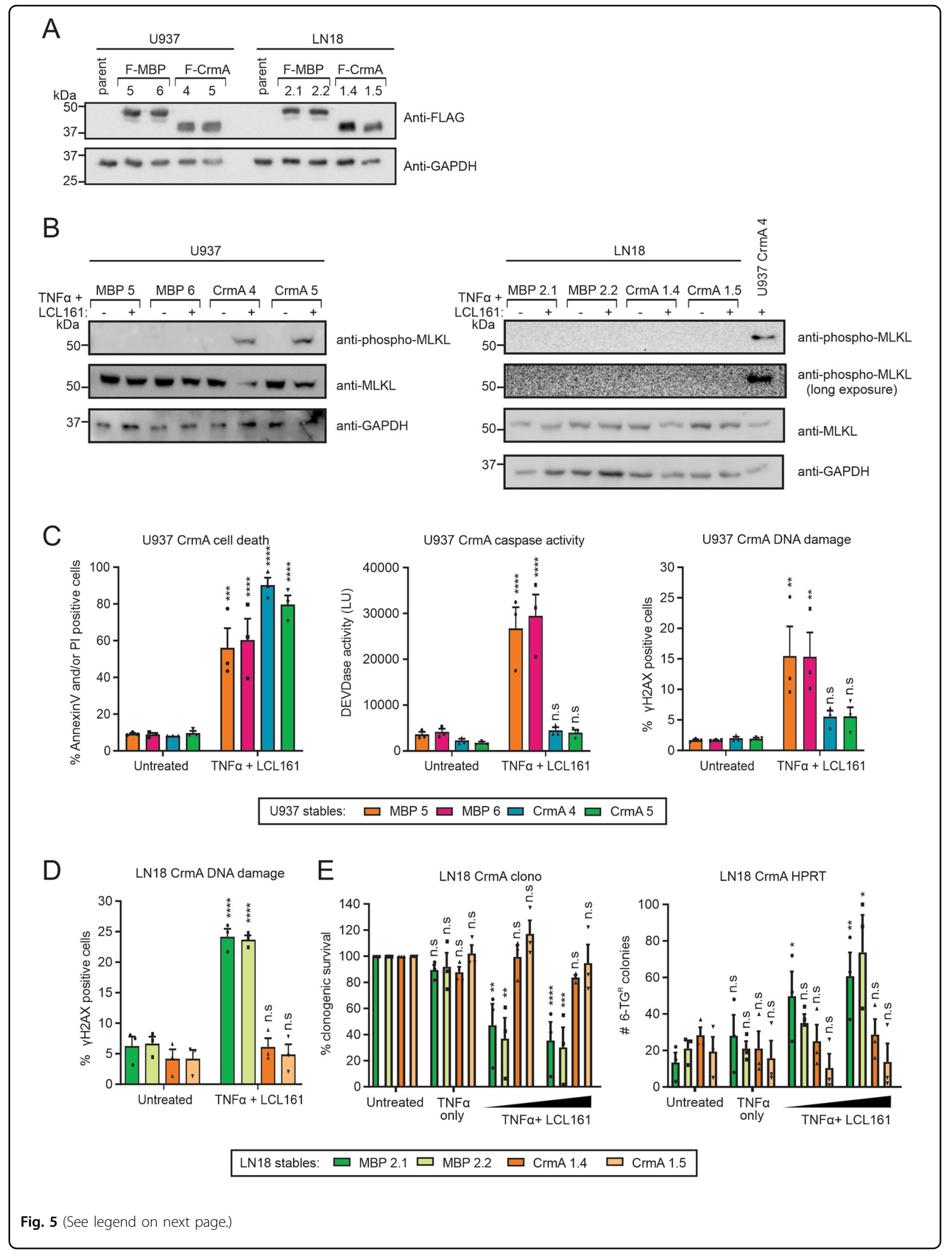


(see figure on previous page)

Fig. 5 CrmA expression prevents mutagenesis following TNFa and LCL161 co-treatment. a Stable U937 or LN18 transfectants expressing FLAG tagged MBP or CrmA were lysed and immunoblotted. b Lysates were generated from cells untreated (-) or treated (+) with $1 \mathrm{ng} / \mathrm{ml}(U 937)$ or $10 \mathrm{pg} / \mathrm{ml}$ (LN18) TNFa plus $10 \mu \mathrm{M} \mathrm{LCL161} \mathrm{for} 8 \mathrm{~h}$, and immunoblotting performed to determine phosphorylated and total levels of MLKL. c U937 transfectants were treated with $1 \mathrm{ng} / \mathrm{ml}$ TNFa plus $10 \mu \mathrm{M} \mathrm{LCL} 161$ then cell death assessed after $24 \mathrm{~h}$ (left panel). Caspase activity (middle panel) and DNA damage (right panel) was determined after $6 \mathrm{~h}$. LN18 transfectants were treated with $10 \mathrm{pg} / \mathrm{ml}$ TNFa plus LCL161 (10 $\mu \mathrm{M}$ for panel d, 1 or $3 \mu \mathrm{M}$ for panel e) then d DNA damage determined after $6 \mathrm{~h}$. e After $24 \mathrm{~h}$, surviving cells were assessed for clonogenic viability (left panel) and their ability to grow and form colonies in 6-TG (right panel). Two-way ANOVA with Sidak post-tests were used to estimate the probability that random chance accounted for the differences observed between untreated and treated cells $\left({ }^{*} p<0.05\right.$; ${ }^{* *} p<0.01 ;{ }^{* * *} p<0.001$; ${ }^{* * *} p<0.0001$; ${ }^{n 5} p>0.05$; data represent mean \pm SEM from three independent biological replicates).

activation of executioner caspases. This adds to prior research ascribing mutagenic and oncogenic properties to sublethal apoptotic caspase signaling ${ }^{7,8,11,51,52}$, and assigns for the first time mutagenic potential to Smac mimetics. TNF $\alpha$ and Smac mimetic co-treatment plus QVD or CrmA expression killed U937 or MEF cells without activating caspases and this was dependent on the activity or expression RIPK1, RIPK3 or MLKL, confirming classical necroptotic death. Cells undergoing necroptosis lacked $\gamma \mathrm{H} 2 \mathrm{AX}$ (and therefore DNA damage) and surviving cells did not acquire HPRT mutations, attributing the mutagenicity of Smac mimetics to their caspase-activating function. Although drug-treated surviving cells were cultured for a standard 7 day phenotypic lag period prior to incubation in 6-TG to ensure stable expression of a HPRT mutant phenotype ${ }^{36}$, it is possible that a shorter incubation time could potentially reveal some HPRT mutant clones emerging after a necroptotic stimulus, given that these clones maintained colony forming potential. LN18 cells that were engineered to express RIPK3 became sensitive to necroptotic stimuli and failed to acquire HPRT mutations under caspase-independent conditions that enabled MLKL phosphorylation, whereas mutations ensued in caspase-proficient, MLKLindependent conditions. Coupled with the inability of a constitutively active MLKL mutant to provoke DNA damage, our data demonstrate that caspase-independent necroptotic cell death avoids the DNA damage and mutations associated with signaling pathways that activate caspases. These results support the onco-protective effects of caspase-independent modes of cell death ${ }^{53}$.

The clinical use of Smac mimetics is currently being evaluated and their ability to mutate cells in vivo, and therefore impact the risk of therapy-related cancers, remains to be determined. Our data imply that the mutagenic potential of Smac mimetics to cells of different lineages within various organs will hinge on the local concentration of TNF $\alpha$ and the status of caspase- 8 and RIPK3 within the cells, as these factors would be expected to influence whether cells respond to IAP antagonism by activating potentially mutagenic apoptotic signaling or non-mutagenic necroptotic signaling. Pro-inflammatory cytokines are released upon activation of necroptotic proteins or through stimulation of noncanonical $\mathrm{NF}_{\mathrm{K}} \mathrm{B}$ pathways by stabilization of NIK upon Smac mimetic treatment ${ }^{54-56}$. For instance, RIPK1 expression can modulate $\mathrm{TNF} \alpha$ production by $\mathrm{NF} \mathrm{BB}$ dependent and independent pathways ${ }^{57,58}$, and the secretion of chemokines and other immunoregulatory molecules occur in a RIPK3- and MLKL-dependent manner during necroptosis $^{59,60}$. Therefore, it may be possible for the autocrine TNF $\alpha$ produced by circulating or tumor-infiltrating myeloid cells ${ }^{61,62}$, for example, in patients treated with Smac mimetics to cooperate with the drug to trigger sublethal apoptotic signaling in noncancerous cells, which may be mutagenic and potentially could lead to oncogenic transformation.

Encouragingly, we did not observe DNA damage upon induced expression of activated MLKL suggesting future drugs that directly activate MLKL (without the ability to also activate caspases or raise TNF $\alpha$ levels, as Smac mimetics do) may harbor low mutagenic risk. Mechanisms that enable cells to withstand modest levels of MLKL activation have been characterized offering evidence to support our findings ${ }^{42,59,63-65}$. Direct MLKL activation may also avoid the potential accumulation of RIPK3driven oxidative stress upon stimulation of the necrosome as has been reported following TNF $\alpha / \mathrm{Smac}$ mimetic treatment, pathogen infection or necrosome formation ${ }^{66-70}$. As we did not detect evidence of DNA damage (or mutations) in necroptotic cells, we speculate that any RIPK3-dependent oxidative stress formed had no genotoxic effect.

In conclusion, this study provides hope for the safety of direct necroptosis inducing anti-cancer agents developed in the future as they may reduce the risk of therapyrelated second cancers in patients whose first malignancies are cured using these therapies. Further work will be needed to determine whether Smac mimetics are nonmutagenic in vivo, or whether they can cooperate with TNF $\alpha$ to trigger apoptotic signaling, potentially rendering them mutagenic and possibly oncogenic in vivo.

\section{Acknowledgements}

We thank James Murphy for cell lines and the MLKL antibody, Marco Herold and Hamsa Puthalakath for reagents and assistance with CRISPR gene editing, and the LIMS Bioimaging Platform. This study was funded by a Cancer Council 
Victoria Postdoctoral Fellowship to M.A.M., a grant from The Kids' Cancer Project and a Grant-in-Aid from the Cancer Council Victoria awarded to C.J.H.

\section{Conflict of interest}

The authors declare that they have no conflict of interest.

\section{Publisher's note}

Springer Nature remains neutral with regard to jurisdictional claims in published maps and institutional affiliations.

Supplementary Information accompanies this paper at (https://doi.org/ 10.1038/s41419-020-02879-y).

Received: 18 May 2020 Revised: 4 August 2020 Accepted: 4 August 2020 Published online: 13 August 2020

\section{References}

1. Matt, S. \& Hofmann, T. G. The DNA damage-induced cell death response: a roadmap to kill cancer cells. Cell Mol. Life Sci. 73, 2829-2850 (2016).

2. Merino, D. et al. BH3-mimetic drugs: blazing the trail for new cancer medicines. Cancer Cell 34, 879-891 (2018).

3. Yuan, X. et al. Developing TRAIL/TRAIL death receptor-based cancer therapies. Cancer Metastasis Rev. 37, 733-748 (2018).

4. Alexandrov, L. B. et al. Signatures of mutational processes in human cancer Nature 500, 415-421 (2013).

5. Friedman, D. L. et al. Subsequent neoplasms in 5-year survivors of childhood cancer: the Childhood Cancer Survivor Study. J. Natl Cancer Inst. 102 1083-1095 (2010).

6. Bhatia, S. Genetic variation as a modifier of association between therapeutic exposure and subsequent malignant neoplasms in cancer survivors. Cancer 121, 648-663 (2015). Epub 2015 Feb 19.

7. Lovric, M. M. \& Hawkins, C. J. TRAlL treatment provokes mutations in surviving cells. Oncogene 29, 5048-5060 (2010).

8. Ichim, G. et al. Limited mitochondrial permeabilization causes DNA damage and genomic instability in the absence of cell death. Mol. Cell 57, 860-872 (2015).

9. Tang, H. L. et al. Cell survival, DNA damage, and oncogenic transformation after a transient and reversible apoptotic response. Mol. Biol. Celll 23 2240-2252 (2012)

10. Sakahira, H., Enari, M. \& Nagata, S. Cleavage of CAD inhibitor in CAD activation and DNA degradation during apoptosis. Nature 391, 96-99 (1998).

11. Miles, M. A. \& Hawkins, C. J. Executioner caspases and CAD are essential for mutagenesis induced by TRAll or vincristine. Cell Death Dis. 8, e3062 (2017).

12. Larsen, B. D. \& Sorensen, C. S. The caspase-activated DNase: apoptosis and beyond. FEBS J. 284, 1160-1170 (2017).

13. Ichim, G. \& Tait, S. W. A fate worse than death: apoptosis as an oncogenic process. Nat. Rev. Cancer 16, 539-548 (2016). Epub 2016 Jul 31.

14. Mohamed, M. S., Bishr, M. K., Almutairi, F. M. \& Ali, A. G. Inhibitors of apoptosis: clinical implications in cancer. Apoptosis 22, 1487-1509 (2017).

15. Rathore, R., McCallum, J. E., Varghese, E., Florea, A.-M. \& Büsselberg, D. Overcoming chemotherapy drug resistance by targeting inhibitors of apoptosis proteins (IAPs). Apoptosis 22, 898-919 (2017).

16. Morrish, E., Brumatti, G. \& Silke, J. Future therapeutic directions for Smacmimetics. Cells 9, 406 (2020).

17. Amaravadi, R. K. et al. A phase I study of the SMAC-mimetic birinapant in adults with refractory solid tumors or lymphoma. Mol. Cancer Ther. 14, 2569-2575 (2015).

18. Infante, J. R. et al. Phase I dose-escalation study of LCL161, an oral inhibitor of apoptosis proteins inhibitor, in patients with advanced solid tumorsJ. Clin. Oncol. 32, 3103-3110 (2014).

19. DiPersio, J. F. et al. Oral Debio1143 (AT406), an antagonist of inhibitor of apoptosis proteins, combined with daunorubicin and cytarabine in patients with poor-risk acute myeloid leukemia-results of a phase I dose-escalation study. Clin. Lymphoma Myeloma Leuk. 15, 443-449 (2015).

20. Flygare, J. A. Discovery of a potent small-molecule antagonist of inhibitor of apoptosis (IAP) proteins and clinical candidate for the treatment of cancer (GDC-0152). J. Med. Chem. 55, 4101-4113 (2012).
21. Bertrand, M. J. et al. CIAP1 and CIAP2 facilitate cancer cell survival by functioning as E3 ligases that promote RIP1 ubiquitination. Mol. Cell 30, 689-700 (2008).

22. Petersen, S. L. et al. Autocrine TNFalpha signaling renders human cancer cells susceptible to Smac-mimetic-induced apoptosis. Cancer Cell 12, 445-456 (2007).

23. Shiozaki, E. N. et al. Mechanism of XIAP-mediated inhibition of caspase-9. Mol. Cell 11, 519-527 (2003).

24. Suzuki, Y., Nakabayashi, Y., Nakata, K. Reed, J. C. \& Takahashi, R . X-linked inhibitor of apoptosis protein (XIAP) inhibits caspase-3 and -7 in distinct modes. J. Biol. Chem. 276, 57058-27063 (2001).

25. Dondelinger, $Y$. et al. RIPK3 contributes to TNFR1-mediated RIPK1 kinasedependent apoptosis in conditions of CIAP1/2 depletion or TAK1 kinase inhibition. Cell Death Differ. 20, 1381-1392 (2013).

26. Cai, Z. et al. Plasma membrane translocation of trimerized MLKL protein is required for TNF-induced necroptosis. Nat. Cell Biol. 16, 55-65 (2014).

27. Huang, D. et al. The MLKL channel in necroptosis is an octamer formed by tetramers in a dyadic process. Mol. Cell Biol. 37, e00497-16 (2017).

28. Pasparakis, M. \& Vandenabeele, P. Necroptosis and its role in inflammation. Nature 517, 311-320 (2015)

29. Cho, Y. S. The role of necroptosis in the treatment of diseases. BMB Rep. $\mathbf{5 1}$ 219-224 (2018)

30. Dhuriya, Y. K. \& Sharma, D. Necroptosis: a regulated inflammatory mode of cell death. J. Neuroinflamm. 15, 199 (2018).

31. Stout, J. T. \& Caskey, C. T. HPRT: gene structure, expression, and mutation. Annu Rev. Genet. 19, 127-148 (1985)

32. Miles, M. A., Caruso, S., Baxter, A. A., Poon, I. K. H. \& Hawkins, C. J. Smac mimetics can provoke lytic cell death that is neither apoptotic nor necroptotic Apoptosis 25, 500-518 (2020).

33. Barger, C. J., Branick, C., Chee, L. \& Karpf, A. R. Pan-cancer analyses revea genomic features of FOXM1 overexpression in cancer. Cancers 11, 251 (2019).

34. Shekhar, T. M. et al. Inhibition of BCl-2 or IAP proteins does not provoke mutations in surviving cells. Mutat. Res. 777, 23-32 (2015).

35. Op het Veld, C. W., Van Hees-Stuivenberg, S., Van Zeeland, A. A. \& Jansen, J. G. Effect of nucleotide excision repair on HPRT gene mutations in rodent cells exposed to DNA ethylating agents. Mutagenesis 12, 417-424 (1997).

36. Jacobs, L. \& Demars, R. Quantification of chemical mutagenesis in diploid human fibroblasts: induction of azaguanine-resistant mutants by N-methyl-N'nitro-N-nitrosoguanidine. Mutat. Res. 53, 29-53 (1978).

37. Hain, K. O., Colin, D. J., Rastogi, S., Allan, L. A. \& Clarke, P. R. Prolonged mitotic arrest induces a caspase-dependent DNA damage response at telomeres that determines cell survival. Sci. Rep. 6, 26766 (2016)

38. Miles, M. A., Harris, M. A. \& Hawkins, C. J. Proteasome inhibitors trigger mutations via activation of caspases and CAD, but mutagenesis provoked by the HDAC inhibitors vorinostat and romidepsin is caspase/CAD-independent. Apoptosis 24, 404-413 (2019)

39. Petrie, E. J., Gzabotar, P. E. \& Murphy, J. M. The structural basis of necroptotic cell death signaling. Trends Biochem. Sci. 44, 53-63 (2019).

40. He, S. et al. Receptor interacting protein kinase-3 determines cellular necrotic response to TNF-alpha. Cell 137, 1100-1111 (2009).

41. Yan, B. et al. Discovery of a new class of highly potent necroptosis inhibitors targeting the mixed lineage kinase domain-like protein. Chem. Commun. $\mathbf{5 3}$ 3637-3640 (2017)

42. Sun, L. et al. Mixed lineage kinase domain-like protein mediates necrosis signaling downstream of RIP3 kinase. Cell 148, 213-227 (2012).

43. Arnež, K.H. et al. Analysis of the N-terminal region of human MLKL, as well as two distinct MLKL isoforms, reveals new insights into necroptotic cell death. Biosci. Rep. 36, e00291 (2015).

44. Galluzzi, L. et al. Molecular mechanisms of cisplatin resistance. Oncogene $\mathbf{3 1}$ 1869-1883 (2012)

45. Olive, P. L. \& Banath, J. P. Kinetics of H2AX phosphorylation after exposure to cisplatin. Cytom. B Clin. Cytom. 76, 79-90 (2009).

46. Bloomer, D. T. et al. CrmA orthologs from diverse poxviruses potently inhibit caspases- 1 and -8 , yet cleavage site mutagenesis frequently produces caspase1-specific variants. Biochem. J. 476, 1335-1357 (2019).

47. Huang, $X$. et al. Bypassing drug resistance by triggering necroptosis: recent advances in mechanisms and its therapeutic exploitation in leukemia. J. Exp. Clin. Cancer Res. 37, 310 (2018).

48. Johnson, B. E. et al. Mutational analysis reveals the origin and therapy-driven evolution of recurrent glioma. Science 343, 189-193 (2014).

49. Ding, L. et al. Clonal evolution in relapsed acute myeloid leukaemia revealed by whole-genome sequencing. Nature 481, 506-510 (2012). 
50. Miles, M. A. \& Hawkins, C. J. Mutagenic assessment of chemotherapy and Smac mimetic drugs in cells with defective DNA damage response pathways. Sci. Rep. 8, 14421 (2018).

51. Miles, M. A., Shekhar, T. M., Hall, N. E. \& Hawkins, C. J. TRAlL causes deletions at the HPRT and TK1 loci of clonogenically competent cells. Mutat. Res. 787, 15-31 (2016).

52. Orth, J. D., Loewer, A., Lahav, G. \& Mitchison, T. J. Prolonged mitotic arrest triggers partial activation of apoptosis, resulting in DNA damage and p53 induction. Mol. Biol. Cell 23, 567-576 (2012).

53. Cao, K. \& Tait, S. W. G. Apoptosis and cancer: force awakens, phantom menace, or both? Int. Rev. Cell Mol. Biol. 337, 135-152 (2018).

54. Zhu, K et al. Necroptosis promotes cell-autonomous activation of proinflammatory cytokine gene expression. Cell Death Dis. 9, 500 (2018).

55. Silke, J. \& Brink, R. Regulation of TNFRSF and innate immune signalling complexes by TRAFs and CIAPs. Cell Death Differ. 17, 35-45 (2010).

56. Tchoghandjian, A., Jennewein, C., Eckhardt, I., Rajalingam, K. \& Fulda, S. Identification of non-canonical NF-kappaB signaling as a critical mediator of Smac mimetic-stimulated migration and invasion of glioblastoma cells. Cell Death Dis. 4, e564 (2013).

57. Christofferson, D. E., Li, Y. \& Yuan, J. Control of life-or-death decisions by RIP1 kinase. Annu. Rev. Physiol. 76, 129-150 (2014).

58. Christofferson, D. E. et al. A novel role for RIP1 kinase in mediating TNFalpha production. Cell Death Dis. 3, e320 (2012).

59. Gong, Y. N. et al. ESCRT-III acts downstream of MLKL to regulate necroptotic cell death and its consequences. Cell 169, 286-300 e216 (2017).

60. Orozco, S. L. et al. RIPK3 activation leads to cytokine synthesis that continues after loss of cell membrane integrity. Cell Rep. 28, 2275-2287.e2275 (2019).
61. Shekhar, T. M. et al. Smac mimetics LCL161 and GDC-0152 inhibit osteosarcoma growth and metastasis in mice. BMC Cancer 19, 924 (2019).

62. Lecis, D. et al. Smac mimetics induce inflammation and necrotic tumour cell death by modulating macrophage activity. Cell Death Dis. 4, e920 (2013).

63. Gong, Y. N., Guy, C., Crawford, J. C. \& Green, D. R. Biological events and molecular signaling following MLKL activation during necroptosis. Cell Cycle 16, 1748-1760 (2017).

64. Yoon, S., Kovalenko, A., Bogdanov, K. \& Wallach, D. MLKL, the protein that mediates necroptosis, also regulates endosomal trafficking and extracellular vesicle generation. Immunity 47, 51-65.e57 (2017).

65. Zargarian, S. et al. Phosphatidylserine externalization, "necroptotic bodies" release, and phagocytosis during necroptosis. PLOS Biol. 15 e2002711 (2017).

66. Yang, Z. et al. RIP3 targets pyruvate dehydrogenase complex to increase aerobic respiration in TNF-induced necroptosis. Nat. Cell Biol. 20, 186-197 (2018).

67. Schenk, B. \& Fulda, S. Reactive oxygen species regulate Smac mimetic/ TNFalpha-induced necroptotic signaling and cell death. Oncogene 34, 5796-5806 (2015).

68. Zhang, Y. et al. RIP1 autophosphorylation is promoted by mitochondrial ROS and is essential for RIP3 recruitment into necrosome. Nat. Commun. 8, 14329 (2017).

69. Cho, Y. S. et al. Phosphorylation-driven assembly of the RIP1-RIP3 complex regulates programmed necrosis and virus-induced inflammation. Cell 137 1112-1123 (2009)

70. Barbosa, L. A. et al. RIPK1-RIPK3-MLKL-associated necroptosis drives leishmania infantum killing in neutrophils. Front. Immunol. 9, 1818 (2018). 